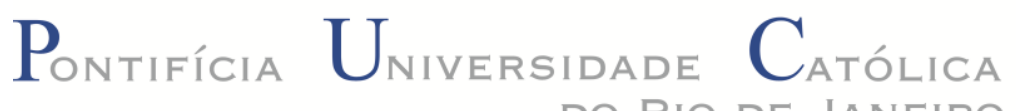

DO RIO DE JANEIRO

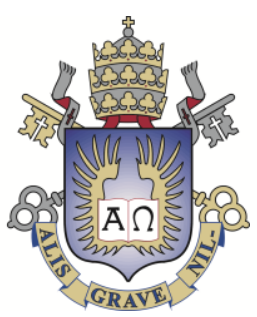

Edson Borges da Silva Neto

\title{
BIOSSORÇÃO DE Co(II) E Ni(II) CONTIDOS EM SOLUÇÕES AQUOSAS UTILIZANDO A CEPA
}

Rhodococcus ruber

Dissertação de Mestrado

Dissertação apresentada como requisito parcial para obtenção do título de Mestre pelo Programa de Pós-Graduação em Engenharia dos Materiais e de Processos Químicos e Metalúrgicos do Departamento de Engenharia de Materiais da PUCRio.

Orientador: Prof. Maurício Leonardo Torem Co-Orientadora: Iranildes Daniel dos Santos 
Pontifícia Universidade Católica $_{\text {a }}$

Edson Borges da Silva Neto

\title{
BIOSSORÇÃO DE Co(II) E Ni(II) CONTIDOS EM SOLUÇÕES AQUOSAS UTILIZANDO A CEPA
}

Rhodococcus ruber

\begin{abstract}
Dissertação apresentada como requisito parcial para obtenção do grau de Mestre pelo Programa de PósGraduação em Engenharia de Materiais e de Processos Químicos e Metalúrgicos do Departamento de Engenharia de Materiais (DEMa) da PUC-Rio. Aprovada pela Comissão Examinadora abaixo assinada.
\end{abstract}

\section{Prof. Maurício Leonardo Torem} Orientador

Departamento de Engenharia de Materiais - PUC-Rio

Profa. Iranildes Daniel dos Santos Departamento de Engenharia de Materiais - PUC-Rio

Prof. Eduardo de Albuquerque Brocchi Departamento de Engenharia de Materiais - PUC-Rio

Profa. Denise Maria Mano Pessôa Departamento de Engenharia Civil - PUC-Rio

Profa. Mônica Regina da Costa Marques Instituto de Química - UERJ

Prof. José Eugênio Leal Coordenador Setorial do Centro Técnico Científico - PUC-Rio

Rio de Janeiro, 18 de agosto de 2011. 
Todos os direitos reservados. É proibida a reprodução total ou parcial do trabalho sem autorização da universidade, do autor e do orientador.

Edson Borges da Silva Neto

Graduou-se em Engenharia Agronômica pela Universidade Federal Rural de Pernambuco em 2004. Atuou por seis anos no mercado de trabalho em multinacionais do segmento de insumos agrícolas e como consultor técnico na área ambiental. Sendo sua experiência na área acadêmica sempre relacionando o uso de microorganismos com potencial biotecnológico e aplicabilidade ambiental.

Ficha Catalográfica

Silva Neto, Edson Borges da

Biossorção de Co(II) e Ni(II) contidos em soluções aquosas utilizando a cepa Rhodococcus ruber / Edson Borges da Silva Neto ; orientadores: Maurício Leonardo Torem, Iranildes Daniel dos Santos. - 2011.

136 f. : il. (color.) ; $30 \mathrm{~cm}$

Dissertação (mestrado)-Pontifícia Universidade Católica do Rio de Janeiro, Departamento de Engenharia de Materiais, 2011.

Inclui bibliografia

1. Engenharia de materiais - Teses. 2. Remoção. 3. Bactéria. 4. Metais pesados. 5. Tecnologia ambiental. 6. Biorremediação. I. Torem, Maurício Leonardo. II. Santos, Iranildes Daniel dos. III. Pontifícia Universidade Católica do Rio de Janeiro. Departamento de Engenharia de Materiais. IV. Título. 
Dedico a toda minha família, em especial aos meus pais Celia e Edmilson; e meu irmão Thiago que me acompanharam sempre na minha luta por um horizonte profissional de sucesso. 


\section{Agradecimentos}

Aos Professores orientadores Professor Maurício Leonardo Torem e Dra. Iranildes Daniel dos Santos pela oportunidade para a realização deste curso e confiança me dando total independência para execução dos trabalhos acadêmicos relacionados a dissertação.

A Dra. Gabriela Alejandra Huáman Pino pelas considerações e aporte indispensáveis para condução e resolução dos problemas e soluções relacionadas aos trabalhos experimentais.

Ao CNPq, FAPERJ e a PUC-Rio pela concessão de bolsa de estudos, auxílio financeiro e de isenção de taxas do curso de mestrado, respectivamente.

Á Professora Dra. Denise Maria Mano Pessôa por toda sua dedicação, amizade, sugestões e suporte não só profissionais, mas também deixo expresso meu agradecimento por ter me ajudado em situações pessoais nos dias de hoje raramente encontramos pessoas assim, muito obrigado!

Agradecimento ao Departamento de Química da PUC-Rio, ao Professor Doutor Reinaldo de Campos Calixto e em especial ao técnico Rodrigo Gonçalves e demais auxiliares do laboratório pela ajuda e paciência na execução das análises de amostras em Absorção Atômica. 
Ao Professor Júlio Carlos Afonso do Instituto de Química da UFRJ pela concessão das análises de Espectrometria de Infravermelho. A professora Mônica Marques do Instituto de Química da UERJ também pelas análises de Infravermelho.

Agradecimento mais do que especial a Doutora Neusa de Lima Nogueira e a Bióloga e Laboratorista Mônica Lanzoni Rossi do CENA/USP por todo profissionalismo, competência e total dedicação a vida acadêmica, ficando muito grato pelo preparo e as análises das amostras de MEV e MET realizadas. Não deixando de citar o agradecimento, também especial, ao Doutor Elliott Watanabe Kitajima, Coordenador do Núcleo de Apoio à Pesquisa em Microscopia Eletrônica Aplicada à Pesquisa Agropecuária (NAP/MEPA) na ESALQ/USP, por ceder horários nos equipamentos de Microscopia Eletrônica para realização das análises desta dissertação.

Aos professores da PUC-Rio, lotados em diversos departamentos onde obtive conhecimentos para me tornar mestre em Engenharia, na área especifica de Processos e Meio Ambiente. Neste período pude concretizar uma formação sólida e diferenciada para atuar na Engenharia Ambiental.

Um agradecimento especial a todos os colegas da Casa XXI que me acompanharam nesta trajetória. Meus sinceros agradecimentos aos grandes amigos Abiatar Picoli Cardoso e Lórgio Valdiviezo por sua amizade, pelos cafés dos vários fins de tarde, pela ajuda indispensável e providencial nas horas mais difíceis, principalmente para a confecção dos resultados desta dissertação.

Finalmente não posso deixar de citar a todos familiares e amigos que torcem pela minha formação na busca por um caminho profissional brilhante num futuro próximo, prometo não decepcioná-los! 


\section{Resumo}

Silva Neto, Edson Borges da; Torem, Maurício Leonardo. Biossorção de $\mathrm{Co}$ (II) e Ni(II) Contidos em Soluções Aquosas Utilizando a Cepa Rhodococcus ruber. Rio de Janeiro, 2011. 136p. Dissertação de Mestrado - Departamento de Engenharia de Materiais, Pontifícia Universidade Católica do Rio de Janeiro.

A água, recurso natural imprescindível, encontra-se cada vez mais escassa e imprópria para o consumo humano. A biossorção é uma tecnologia que utiliza diversos tipos de biomassas, entre elas microrganismos não patogênicos, como Rhodococcus ruber. O objetivo deste trabalho visa a remoção de $\mathrm{Co}(\mathrm{II})$ e $\mathrm{Ni}(\mathrm{II})$ presentes em soluções aquosas utilizando $R$. ruber. Estudos microbiológicos, ensaios de biossorção em batelada foram realizados para testar a eficiência da biomassa nos metais pesados Co(II) e Ni(II) através dos parâmetros: $\mathrm{pH}$, concentração de biomassa e concentração do metal. $A$ cinética da reação foi estudada e as isotermas confeccionadas. Espectrometria no infravermelho potencial zeta e microscopia eletrônica foram técnicas utilizadas para conhecimento dos elementos envolvidos no processo biossortivo. Uma remoção de $44 \%$ de $\mathrm{Co}(\mathrm{II})$ e $36,6 \%$ de $\mathrm{Ni}(\mathrm{II})$ da solução foi obtida em pH 6 após 180 minutos de contato em solução contendo $3 \mathrm{~g} \cdot \mathrm{L}^{-1}$ de biomassa e $30 \mathrm{mg} \cdot \mathrm{L}^{-1}$ de metal. A partir da concentração de biomassa de $3 \mathrm{~g} . \mathrm{L}^{1}$, observou-se redução na remoção do metal tanto para Co(II) quanto para $\mathrm{Ni}(\mathrm{II})$. A isoterma que melhor se ajustou no estudo em questão foi Freundlich para os dois metais. O modelo de pseudo-segunda ordem foi o mais indicado nos estudos cinéticos realizados para os íons Co(II) e Ni(II). Quando avaliados os melhores parâmetros, não foram incrementados grandes graus de remoção utilizando mais uma ou duas fases de biomassa nova na solução. O tratamento da biomassa com $\mathrm{NaOH}$ foi o mais eficiente elevando a remoção para $97 \%$ de Co(II) e $89,1 \%$ de $\mathrm{Ni}(\mathrm{II})$.

\section{Palavras-chave}

Remoção; Bactéria; Metais Pesados; Tecnologia Ambiental; Biorremediação. 


\section{Abstract}

Silva Neto, Edson Borges da; Torem, Maurício Leonardo (Advisor). Biosorption of $\mathrm{Co}$ (II) and $\mathrm{Ni}$ (II) from Aqueous Solutions Using Rhodococcus ruber Strain. Rio de Janeiro, 2011. 136p. MSc. Dissertation - Departamento de Engenharia de Materiais, Pontifícia Universidade Católica do Rio de Janeiro.

Water, essential natural resource, is becoming increasingly scarce and unfit for human consumption. The biosorption is a technology that uses various types of biomass, including nonpathogenic microorganism suchs Rhodococcus rubber. This study aims at the removal of $\mathrm{Co}(\mathrm{II})$ and $\mathrm{Ni}(\mathrm{II})$ present in aqueous solutions using $R$. rubber. Microbiological studies, batch biosorption test were conducted to test the efficiency of biomass in heavy metals $\mathrm{Co}$ (II) and $\mathrm{Ni}(\mathrm{II})$ through the $\mathrm{pH}$, biomass concentration and concentration of the metal. The reaction kinetics was studie and the isotherms made. Zeta potencial, infrared spectrometry and electron microscopy techniques were used for knowledge of the elements involved microscopy techniques were used for knowledge of the elements involved in the process biosorption. A $44 \%$ removal of Co(II) and $36.6 \% \mathrm{Ni}(\mathrm{II})$ solution at $\mathrm{pH} 6$ was obtained after 180 minutes of contact in a solution containing $3 \mathrm{~g} \cdot \mathrm{L}^{-1}$ of biomass and $30 \mathrm{mg} \cdot \mathrm{L}^{-1}$ of metal solution. From the concentration of biomass $3 \mathrm{~g} \cdot \mathrm{L}^{-1}$, there was a reduction in metal removal for both $\mathrm{Co}(\mathrm{II})$ and for $\mathrm{Ni}(\mathrm{II})$. The isotherm best fit in the current study was Freundlich for the two metals. The model of pseudo-second order was the best in the kinetic studies performed for the $\mathrm{Co}(\mathrm{II})$ and $\mathrm{Ni}(\mathrm{II})$. When considering the best parameters, therewere increased levels of large removal using one or two phases of new biomass in the solution. Treatment of biomass with $\mathrm{NaOH}$ was the most efficient removal rising to $97 \%$ of $\mathrm{Co}(\mathrm{II})$ and $89.1 \% \mathrm{Ni}(\mathrm{II})$.

\section{Keywords}

Removal; Bacteria; Heavy Metals; Environmental Technology; Bioremediation. 


\section{Sumário}

1 Introdução 20

2 Objetivos e Relevância do Trabalho 22

2.1. Objetivo Geral 22

2.2. Objetivos Específicos 23

3 Revisão Bibliográfica $\quad 24$

3.1. Metais Pesados ou Metais Tóxicos $\quad 24$

3.1.1. Cobalto 26

3.1.2. Níquel 27

3.2. Métodos Convencionais de Remoção de Metais Pesados ou Tóxicos 28

3.2.1. Precipitação Química 31

3.2.2. Troca lônica 31

3.2.3. Separação por Membranas $\quad 31$

3.3. Métodos Alternativos de Remoção de Metais Pesados ou Tóxicos 32

3.4. Biossorção 33

3.4.1. Potenciais Biossorventes - Microrganismos 34

3.4.1.1. Fungos 35

$\begin{array}{ll}\text { 3.4.1.2. Leveduras } & 37\end{array}$

3.4.1.3. Bactérias 38

3.4.2. Rhodococcus ruber 39

3.5. Isotermas de Adsorção $\quad 40$

3.5.1. Modelo da Isoterma de Langmuir 43

3.5.2. Modelo da Isoterma de Freundlich $\quad 45$

3.5.3. Modelo da Isoterma de Temkin 46

3.5.4. Modelo da Isoterma de Dubnin-Radushkevich 46

3.6. Variáveis envolvidas no Processo de Biossorção 47

3.6.1. Influência do $\mathrm{pH} \quad 47$

3.6.2. Tempo de Equilíbrio $\quad 48$

3.7. Potencial Zeta 48 
4 Materiais e Métodos $\quad 51$

4.1. Soluções Estoque 51

4.2. Soluções de Íons Metálicos para Biossorção 51

4.3. Microorganismo Utilizado 52

4.3.1. Influência/Efeito do Meio de Cultivo no Crescimento de R. ruber 52

4.3.2. Manutenção, Cultivo e Quantificação de R. ruber 53

4.3.3. Curva de Correlação entre Número de Células x Absorbância 54

4.3.4. Curva de Crescimento de R. ruber 54

4.4. Caracterização da Biomassa de R. ruber 55

4.4.1. Cálculo da Estimativa da Área Superficial Externa da Biomassa 55

4.4.2. Preparo e Análise de Amostras em Microscopia Eletrônica

de Varredura $\quad 55$

4.4.3. Preparo e Análises de Amostras em Microscopia Eletrônica de Transmissão $\quad 57$

4.4.4. Espectrometria por Infravermelho 57

4.4.5. Potencial Zeta 58

4.5. Preparo das Soluções para Testes de Biossorção 59

4.5.1. Efeito do $\mathrm{pH}$

4.5.2. Efeito da Concentração de Biomassa 61

4.5.3. Determinação do Tempo de Equilíbrio 62

4.5.4. Efeito da Concentração Inicial de Metal 63

4.5.5. Tratamento da Biomassa 63

4.5.6. Efeito da Presença de Co(II) e Ni(II) na mesma Solução 64

4.6. Análise Quantitativa da Concentração dos Metais 64

5 Resultados e Discussão 66

5.1. Ensaios Microbiológicos 66

5.1.1. Crescimento de R. ruber em Diferentes Meios de Cultivo 66

5.1.2. Curva de Crescimento de R. ruber 67

5.1.3. Curva de Correlação entre Absorbância e Número de Células 69

5.1.4. Área Superficial Externa de R. ruber 71

5.2. Caracterização do Biossorvente - R. ruber 72

5.2.1. Microscopia Ótica de Luz - R. ruber 72

5.2.2. Microscopia Eletrônica de Varredura - R. ruber 73

5.2.3. Microscopia Eletrônica de Transmissão - R. ruber 75

5.3. Análises de Espectroscopia no Infravermelho 77 
5.3.1. Análises de Espectroscopia no Infravermelho -

$\begin{array}{ll}R . & \text { ruber sem Tratamento } \\ \end{array}$

5.3.2. Análises de Espectroscopia no Infravermelho -

R. ruber Tratada com $\mathrm{NaOH} \quad 79$

5.4. Determinação do Potencial - Estudos Eletrocinéticos 82

5.5. Testes de Biossorção em Batelada 85

5.5.1. Influência do pH na Biossorção de Co(II) e Ni(II) 85

5.5.1.1. Influência do pH na Biossorção deCo(II) 87

5.5.1.2. Influência do pH na Biossorção de Ni(II) 89

5.5.2. Influência da Concentração de $R$. ruber na Biossorção

5.5.2.1. Influência da Concentração de R. ruber na Biossorção de Co(II) 90

5.5.2.2. Influência da Concentração de R. ruber na Biossorção de Ni(II) 92

5.5.3. Influência da Concentração Inicial de Metal na Biossorção

de $\mathrm{Co}(\mathrm{II})$ e de $\mathrm{Ni}(\mathrm{II}) \quad 93$

5.5.3.1. Influência da Concentração Inicial de Metal na Biossorção de Co(II) 93

5.5.3.2. Influência da Concentração Inicial de Metal na Biossorção de Ni(II) 94

5.5.4. Influência do Tempo de Contato na Biossorção de Co(II) e de Ni(II) 96

5.5.4.1. Influência do Tempo de Contato na Biossorção de Co(II) 96

5.5.4.2. Influência do Tempo de Contato da Biossorção de Ni(II) 98

5.5.5. Condições Paramétricas Utilizadas nos Experimentos de

Biossorção de Co(II) e de Ni(II) 99

5.5.6. Cinética de Biossorção 99

5.5.6.1. 99

5.5.6.2. Cinética de Biossorção de Co(II) 99

5.5.6.3. Cinética de Biossorção de Ni(II) 101

5.5.7. Isotermas de Biossorção 103

$\begin{array}{ll}\text { 5.5.8. Isotermas de Adsorção } & 106\end{array}$

5.5.9. Biossorção de Co(II) em Diferentes Condições

Experimentais em Relação ao Biossorvente 111

5.5.10. Biossorção de Ni(II) em Diferentes Condições

Experimentais em Relação ao Biossorvente

5.5.11. Biossorção em Sistemas Binários Co(II) e Ni(II) em Diferentes

Condições Experimentais em Relação ao Biossorvente 116

6 Conclusões e Recomendações para Trabalhos Futuros 119

$\begin{array}{lr}\text { 6.1. Conclusões } & 119\end{array}$ 
Referências Bibliográficas 


\section{Lista de Figuras}

Figura 1 - Metodologia empregada nos trabalhos de biossorção realizados

no Laboratório de Tecnologia Mineral e Ambiental da PUC-Rio.

Figura 2 - Classes de Isotermas em função da concentração de equilíbrio.........41

Figura 3 - Esquema ilustrativo dos procedimentos realizados em testes de biossorção em batelada.

Figura 4 - Crescimento de Rhodococcus ruber em diferentes meios de cultura em função do peso seco $\left(\mathrm{g} \cdot \mathrm{L}^{-1}\right)$. 66

Figura 5 - Crescimento da bactéria Rhodococcus ruber em diferentes meios de cultivo - Absorbância versus Tempo (Horas).

Figura 6 - Curva de crescimento da bactéria Rhodococcus ruber-

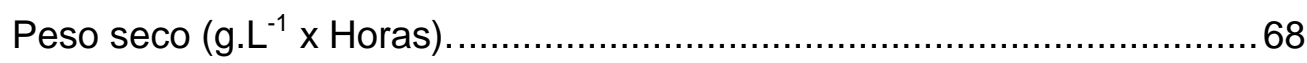

Figura 7 - Curva de crescimento da bactéria Rhodococcus ruber.....................68

Figura 8 - Correlação entre a absorbância e o número de células

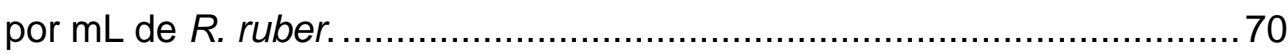

Figura 9 - Microscopia Ótica de Luz de $R$. ruber............................................ 72

Figura 10 - Prancha $1^{*}$ - Imagens de Microscopia Eletrônica de

Varredura da bactéria $R$. ruber e sua interação com metais

Co(II) e Ni(II); A- MEV R. ruber sem tratamento; B - MEV

de R. ruber + Co(II); C - MEV de R. ruber + Ni(II); D - MEV

de $R$. ruber + $\mathrm{Co}(\mathrm{II})+\mathrm{Ni}(\mathrm{II}) ; \mathrm{E}-\mathrm{MEV}$ de $R$. ruber tratada com

$\mathrm{NaOH}$; F - MEV de $R$. ruber tratada com NaOH; G - MEV de

R. ruber tratada com Co(II); H - MEV de R. ruber + Ni(II). ${ }^{*}$ Todas

as imagens possuem a escala de aumento de referência de $2 \mu \mathrm{m}$. ... .74 
Figura 11 - Prancha $2^{*}$ - Imagens de Microscopia Eletrônica de

Transmissão da bactéria $R$. ruber e de sua interação com os íons metálicos $\mathrm{Co}(\mathrm{II})$ e $\mathrm{Ni}(\mathrm{II})$; A- MET $R$. ruber sem tratamento;

$\mathrm{B}-\mathrm{MET}$ de $R$. ruber + Co(II); C - MET de R. ruber + Ni(II); $\mathrm{D}-\mathrm{MET}$ de $R$. ruber + $\mathrm{Co}(\mathrm{II})+\mathrm{Ni}(\mathrm{II}) ; \mathrm{E}-\mathrm{MET}$ de $R$. ruber tratada com $\mathrm{NaOH}$; F - MET de R. ruber tratada com Co(II); $\mathrm{G}-\mathrm{MET}$ de $R$. ruber + Ni(II); $\mathrm{H}-\mathrm{MET}$ de $R$. ruber + Ni(II). * Todas as imagens possuem a escala de aumento a escala de referência que pode ser 1 ou $2 \mu \mathrm{m}$, indicado na respectiva imagem

Figura 12 - Espectros da absorção na região do infravermelho (FTIR):

(A) do biossorvente Rhodococcus ruber sem tratamento; (B) do biossorvente Rhodococcus ruber sem tratamento + Co; (C) do biossorvente Rhodococcus ruber sem tratamento $+\mathrm{Ni}$.

Figura 13 - (A) Espectro no Infravermelho de R. ruber tratada com $\mathrm{NaOH}$ através de espectrometria no infravermelho; (B) Espectro no Infravermelho de $R$. ruber tratada com $\mathrm{NaOH}$ através de espectrometria no infravermelho + Co; (C) Espectro no Infravermelho de $R$. ruber tratada com $\mathrm{NaOH}$ através de espectrometria no infravermelho $+\mathrm{Ni}$.

Figura 14 - Curva de potencial zeta de R. ruber (1 g.L-1) sem tratamento com $\mathrm{NaOH}$ e após interação com os metais Co(II) e Ni(II) (30 mg.L-1).

Figura 15 - Curva de potencial zeta de $R$. ruber $\left(1 \mathrm{~g} \cdot \mathrm{L}^{-1}\right)$ tratada com $\mathrm{NaOH}$ e a interação do biossorvente com os metais $\mathrm{Co}$ (II) e Ni(II) $\left(30 \mathrm{mg}^{-\mathrm{L}^{-1}}\right)$...84 Figura 16 - Remoção e Captação de Co(II) por R. ruber não tratada $\operatorname{com~} \mathrm{NaOH}$, em função da variação do pH (Concentração Inicial do Metal - 50 mg. $\mathrm{L}^{-1}$; Concentração da Biomassa: 1,0 g. $\mathrm{L}^{-1}$;

Velocidade de Agitação: 125 rpm e Tempo de Contato: 3 h).

Figura 17 - Remoção e Captação de Ni(II) por R. ruber não tratada

na variação do parâmetro $\mathrm{pH}$.

(Concentração Inicial do Metal - 50 mg.L $\mathrm{L}^{-1}$;

Concentração da Biomassa: 1,0 g. $\mathrm{L}^{-1}$;

Velocidade de Agitação: 125 rpm e Tempo de Contato: 3 h). 89

Figura 18 - Remoção e Captação de Co(II) por $R$. ruber na variação do parâmetro Concentração de Biomassa

(Concentração Inicial de Metal: 50 mg.L ${ }^{-1}$; pH: 6,0;

Velocidade de Agitação: 125 rpm e Tempo de Contato: 3 h). 
Figura 19 - Remoção e Captação de Ni(II) por R. ruber no processo de biossorção variando o parâmetro Concentração de Biomassa

(Concentração Inicial de Metal: 50 mg.L ${ }^{-1}$; pH: 6,0;

Velocidade de Agitação: 125 rpm e Tempo de Contato: 3 h). 92

Figura 20 - Remoção e Captação de Co(II) por R. ruber no processo de

biossorção utilizando como parâmetro de variação

Concentração Inicial do Metal (pH: 6,0;

Concentração da Biomassa $3 \mathrm{~g} \cdot \mathrm{L}^{-1}$;

Velocidade de Agitação: 125 rpm e Tempo de Contato: 3 h).

Figura 21 - Remoção e Captação de $\mathrm{Ni}(\mathrm{II})$ por $R$. ruber no processo de

biossorção variando o parâmetro

Concentração Inicial do Metal. $(\mathrm{pH}:$ 6,0;

Concentração da Biomassa $3 \mathrm{~g} \cdot \mathrm{L}^{-1}$;

Velocidade de Agitação: 125 rpm e Tempo de Contato: 3 h) 95

Figura 22 - Remoção e Captação de Co(II) por R. ruber no processo de

biossorção variando o parâmetro Tempo de Contato.

(pH: 6,0; Concentração da Biomassa 3 g. $\mathrm{L}^{-1}$;

Concentração Inicial do Metal $30 \mathrm{mg} \cdot \mathrm{L}^{-1} \mathrm{e}$

Velocidade de Agitação: 125 rpm). 96

Figura 23 - Efeito do Tempo de Contato na biossorção de Ni(II)

por R. ruber (pH: 6,0; Concentração da Biomassa 3 g.L $\mathrm{L}^{-1}$;

Concentração Inicial do Metal $30 \mathrm{mg} \cdot \mathrm{L}^{-1} \mathrm{e}$

Velocidade de Agitação: 125 rpm). 98

Figura 24 - Modelo linear de pseudo-primeira ordem da captação

de Co(II) por R. ruber.

Figura 25 - Modelo linear de pseudo-segunda ordem da captação de

Co(II) usando $R$. ruber como biossorvente. 100

Figura 26 - Modelo linear de pseudo-primeira ordem da captação de

$\mathrm{Ni}(\mathrm{II})$ usando $R$. ruber como biossorvente. 101

Figura 27 - Modelo linear de pseudo-segunda ordem da captação de

$\mathrm{Ni}(\mathrm{II})$ usando $R$. ruber como biossorvente. 102

Figura 28 - Captação do íon Co(II) por $R$. ruber em função da concentração

metálica após a biossorção, modelo não linear

(Concentração de Biomassa: 3 g.L L $^{-1}$; pH: 6,0;

Velocidade de Agitação: 125 rpm e Tempo de Contato: 3 h). 104

Figura 29 - Captação do íon Co(II) por R. ruber em função da

concentração metálica após a biossorção, modelo linearizado 
(Concentração de Biomassa: 3 g.L $\mathrm{L}^{-1} ; \mathrm{pH}:$ 6,0;

Velocidade de Agitação: 125 rpm

e Tempo de Contato: $3 \mathrm{~h}$ ). 104

Figura 30 - Captação dos íons Ni(II) por R. ruber em função da

concentração metálica após a biossorção, modelo não linear

(Concentração de Biomassa: 3 g.L $\mathrm{L}^{-1}$; pH: 6,0;

Velocidade de Agitação: 125 rpm e Tempo de Contato: 3 h). 105

Figura 31 - Captação dos íons $\mathrm{Ni}(\mathrm{II})$ por $R$. ruber em função da concentração metálica após a biossorção, modelo linearizado

(Concentração de Biomassa: 3 g.L ${ }^{-1} ; \mathrm{pH}:$ 6,0;

Velocidade de Agitação: 125 rpm e Tempo de Contato: 3 h). 105

Figura 32 - Aplicação da equação de Langmuir para a biossorção de Co(II) por R. ruber. 106

Figura 33 - Aplicação da equação de Freundlich para a biossorção de Co(II) por $R$. ruber. 107

Figura 34 - Aplicação da equação de Tenkim para a biossorção de Co(II) por $R$. ruber. 107

Figura 35 - Aplicação da equação de Dubinin-Radushkevich

para a biossorção de Co(II) por $R$. ruber. 108

Figura 36 - Aplicação da equação de Langmuir para a biossorção

de $\mathrm{Ni}(\mathrm{II})$ por $R$. ruber. 108

Figura 37 - Aplicação da equação de Freundlich para a biossorção de $\mathrm{Ni}(\mathrm{II})$ por $R$. ruber. 109

Figura 38 - Aplicação da equação de Tenkim para a biossorção de $\mathrm{Ni}(\mathrm{II})$ por $R$. ruber. 109

Figura 39 - Aplicação da equação de Dubinin-Radushkevich

para a biossorção de $\mathrm{Ni}(\mathrm{II})$ por $R$. ruber. 110

Figura 40 - Remoção e Captação de Co(II) por $R$. ruber no processo de biossorção em diferentes situações experimentais $(\mathrm{pH}: 6,0$;

Concentração da Biomassa 3 g.L L $^{-1}$;

Concentração Inicial do Metal de $30 \mathrm{mg} \cdot \mathrm{L}^{-1}$,

Tempo de Contato 30 de minutos e

Velocidade de Agitação: 125 rpm). 
Figura 41 - Remoção e Captação de Ni(II) por R. ruber no processo de biossorção em diferentes condições experimentais, conforme descrito nos itens 1 a 4 apresentados $(\mathrm{pH}: 6,0$; Concentração da Biomassa $3 \mathrm{~g} \cdot \mathrm{L}^{-1}$;

Concentração Inicial do Metal de $30 \mathrm{mg} \cdot \mathrm{L}^{-1}$, Tempo de Contato de 30 minutos e Velocidade de Agitação: 125 rpm)

Figura 42 - Remoção e Captação de Co(II) e Ni(II), em sistema

binário de competição, por $R$. ruber no processo de biossorção em diferentes condições experimentais de biomassa e tratamento com $\mathrm{NaOH}(\mathrm{pH}: 6,0$; Concentração da Biomassa $3 \mathrm{~g} \cdot \mathrm{L}^{-1}$; Concentração Inicial do Metal de $30 \mathrm{mg} \cdot \mathrm{L}^{-1}$, Tempo de Contato de 30 minutos e 


\section{Lista de Tabelas}

Tabela 1 - Limites máximos admissíveis em corpos d'águas EPA

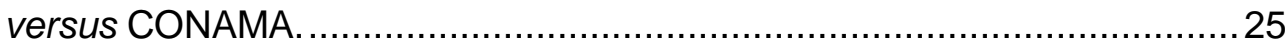

Tabela 2 - Poluentes Inorgânicos e suas origens de deposição........................26

Tabela 4 - Condições empregadas para a determinação da influência do $\mathrm{pH}$ no processo de biossorção. ..................................................... 61

Tabela 5 - Condições empregadas para a determinação da influência da Concentração da Biomassa no processo de biossorção.

Tabela 6 - Condições empregadas na determinação do tempo de equilíbrio. 62

Tabela 7 - Condições empregadas para a determinação da influência da Concentração Inicial de Metal no processo de biossorção.

Tabela 8 - Relação entre diluições, número de células e absorbância de $R$. ruber.

Tabela 9 - Processo de biossorção relacionando diferentes biossorventes, condições de $\mathrm{pH}$ e temperatura; e ajustes da isoterma de Frendluich. 
Paciência e perseverança tem o efeito mágico de fazer as dificuldades desaparecerem e os obstáculos sumirem.

John Quincy Adams 